2019-09

NFV Resource Advertisement and Discovery Protocol for a Distributed NFV Orchestration in a WMN-based Disaster Network

Frick, $\mathrm{G}$

http://hdl.handle.net/10026.1/17148

10.23919/softcom.2019.8903694

2019 International Conference on Software, Telecommunications and Computer Networks (SoftCOM)

IEEE

All content in PEARL is protected by copyright law. Author manuscripts are made available in accordance with publisher policies. Please cite only the published version using the details provided on the item record or document. In the absence of an open licence (e.g. Creative Commons), permissions for further reuse of content should be sought from the publisher or author. 


\title{
NFV Resource Advertisement and Discovery Protocol for a Distributed NFV Orchestration in a WMN-based Disaster Network
}

\author{
Gregor Frick $^{12}$, Auberlin Paguem Tchinda ${ }^{12}$, Ulrich Trick ${ }^{1}$, Armin Lehmann ${ }^{1}$, Bogdan Ghita ${ }^{2}$ \\ ${ }^{1}$ Research Group for Telecommunication networks, Frankfurt University of Applied Sciences, Frankfurt/Main, Germany \\ \{ frick, paguem, trick, lehmann \}@e-technik.org \\ ${ }^{2}$ Centre for Security, Communications and Network Research, University of Plymouth, Plymouth, United Kingdom \\ \{ gregor.frick, auberlin.paguemtchinda, bogdan.ghita \}@plymouth.ac.uk
}

\begin{abstract}
In this publication a Network Function Virtualization (NFV) resource advertisement and discovery protocol for a distributed NFV orchestration in a Wireless Mesh Network (WMN)-based disaster network is proposed. The protocol enables the management of a dynamic NFV infrastructure by automatically assigning the currently required management entity to the nodes in the WMN-based disaster network and thus provides the basis for a distributed orchestration. The protocol is defined using a set of methods and a state machine. By using different scenarios, the state machine and the message flow of the protocol is described in detail.
\end{abstract}

Keywords-Network Function Virtualization, Wireless Mesh Network, Disaster Network, Distributed NFV Orchestration, NFV Resource Advertisement, NFV Resource Discovery

\section{INTRODUCTION}

After natural disasters such as earthquakes, tsunamis, and floods as well as man-made disasters such as persistent power failures, an operating communication infrastructure is assumed as crucial regarding the rescuing of victims in need and the management of rescue teams [1]. Unfortunately, existing telecommunication infrastructures in the affected disaster regions are often damaged or even destroyed.

For providing an emergency communication infrastructure for the rescue helpers and victims, the establishment of a Wireless Mesh Network (WMN)-based disaster network is proposed in [2]. The disaster network is intended to be constructed of battery-supplied wireless outdoor routers, which are deployed in the disaster environment by the first responders and together establish a WMN. The WMN provides the basis for an IP-based network infrastructure. Through the utilisation of the Network Function Virtualisation (NFV) concept [3] in the WMN-based disaster network, a dynamic network service and function provisioning shall be enabled. The provided services include common network services and functionalities (such as DHCP-, DNS-Server, firewalls), communication-related services (such as Multimedia over IP (MoIP), instant message (IM)) and data provisioning services (provided by databases and data storage servers). In addition, other network optimisation aspects such as the overall network availability, reliability and scalability as well as the optimisation of the overall energy-consumption of the network shall be achieved.

A major issue resulting from the integration of NFV into the WMN-based disaster network results from the logically centralised NFV Orchestrator (NFVO) of the architectural NFV framework defined by ETSI in [4]. The NFVO must operate in the WMN, since the breakdown of existing communication infrastructures resulting from the disaster makes a connection to a server hosted in an external network impossible. A WMN node hosting this centralised orchestrator might get damaged, destroyed, or lose its connectivity to large sections of the network due to additional disasters such as aftershocks and outbreaking fires resulting from the environment of a disaster. The loss of the orchestrator is not beneficial, as it will result in losing the possibility to maintain and orchestrate the resources of the NFV infrastructure (NFVI) and thus the provided services. To remove the dependence on a centralised entity, the NFV orchestration needs to be distributed among the WMN nodes.

In order to maintain and orchestrate the NFVI, the distributed NFV orchestration must be aware of all available resources in the network. A major related issue is the likely possibility of dynamic changes in the network topology due to the environment of a disaster. The changes can either be the result of existing nodes being suddenly destroyed due to events such as aftershocks or new routers being deployed in the environment by the rescue helpers. Both cases need to be identified by the distributed NFV orchestration.

The integration of new nodes and their corresponding resources and thus a NFVI-Node is not further explained by the standardisation of ETSI. This might be related to the fact that the NFV framework was mainly designed for telco providers, where the network infrastructure and thus the NFVI is largely static. The integration of new resources into the NFVI via a NFVI-Node therefore requires manual configuration by an administrator. This administration effort cannot be provided in case of the WMN-based disaster network due to the limited technical knowledge of the existing rescue helpers. The operational activities of the rescue helpers only include the deployment of the battery-supplied wireless outdoor routers. For this reason, the integration of new resources provided by a new deployed node as well as the management and formation of the NFVI even in the event of failures must be achieved automatically and largely autonomously by the distributed orchestration. To address these issues, this paper proposes an NFV resource advertisement and discovery protocol to be used towards the establishment and management of a dynamic NFVI.

The protocol enables the distributed orchestration to automatically identify and adapt to the dynamic changes in the network topology of the WMN-based disaster network and thus in the NFVI. Additionally, it supports the distributed orchestration in achieving fault tolerance by enabling a redundant information storage of existing resources in the network if required. While the protocol is designed for usage in a WMN-based disaster network, it could also be utilised in other dynamic wireless networks facing similar limitations.

The protocol is defined using a state machine, which defines the current management functionality each node in the network is providing to the NFVI. Different methods are 
available for the synchronisation and communication between the nodes in the network. These methods are transmitted in the WMN-based disaster network partly via multicast.

The paper is structured as follows. Section II will introduce a distributed NFV orchestration architecture for a WMN-based disaster network since it builds the basis of this publication. In Section III the different states of the protocol state machine are introduced, while in section IV the methods of the protocol and their functionality is presented. In Section $\mathrm{V}$ the general protocol is proposed by describing the behaviour of the state machine at different scenarios. In section VI related publications are investigated. This publication will close with a conclusion in section VII.

\section{ARCHIECTURE FOR A DISTRIBUTED NFV ORCHESTRATION IN A WMN-BASED DISASTER NETWORK}

Major issues and requirements are imposed on the NFV orchestration for the WMN-based disaster network. Primarily, the orchestration requires to have a distributed and decentralised architecture for eliminating the possibility of a single point of failure and providing the basis for a faulttolerant and load balanced orchestration. Additionally, it requires a self-healing architecture to enable a continuous and autonomous orchestration since an architecture based on redundant units is too resource-intensive for the resourcelimited environment resulting from the battery-supplied wireless outdoor routers. Another crucial requirement of the distributed orchestration regarding this publication consists of the automatic resource integration, which is also required for supporting a scalable and distributed infrastructure.

In order to cope with these and other requirements, an architecture for a distributed NFV architecture in a WMNbased disaster network is proposed. The architecture consists of the clustering of the NFVI, with each NFVI-Cluster defining an area of responsibility for a Cluster-NFVO (CNFVO). According to [5], clustering is a common technique for introducing hierarchy and therefore scalability into a network. Additionally, according to [6], clustering in wireless networks can decrease the complexity regarding a required coordination and synchronisation in the network.

The architecture for the distributed NFV orchestration is shown in figure 1 and is based on multiple logical layers. Each layer is providing a specific functionality to the architecture. An initial version of the architecture was published in [7], but an updated and adjusted architecture is presented in the following by describing the functionality of each layer.

\section{A. Clustered WMN-based NFVI Layer}

The Clustered WMN-based NFVI Layer consists of the actual battery-supplied wireless outdoor routers and is realising the actual network architecture of the WMN-based disaster network and thus the NFVI. The network architecture consists of the clustering of the network. Through the usage of different Wi-Fi channels in each cluster the characteristics of the MAC protocol, the exposed node problem and the hidden terminal problem occurring in a single-radio network are compensated. This should enable a sufficient bandwidth for a multi-hop communication which is mandatory for providing a suitable MoIP-Communication for the rescue helpers and victims.

To achieve the connections between the different clusters and establish the overall WMN, cluster gateways are located at the border of the clusters. Cluster gateways are like ordinary

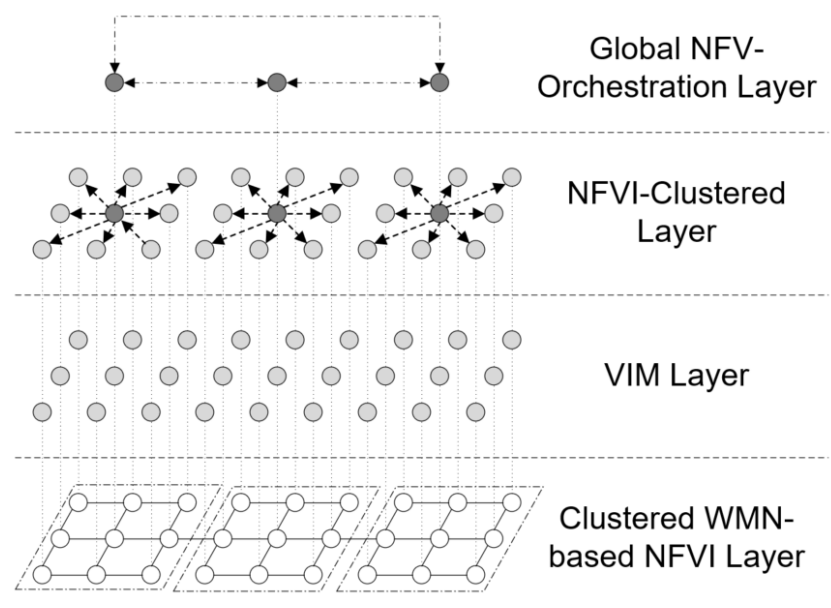

$\begin{array}{llll}\bigcirc & \text { WMN-Node } & \bigcirc & \text { VIM } \\ - & \text { WMN-Connection } & \bigcirc & \text { Cluster-NFVO } \\ \ldots & \text { WMN-Clusters } & -\rightarrow & \text { Responsibility } \\ \ldots & \text { Logical relation } & -\rightarrow & \text { Intra-Orchestration }\end{array}$

Fig. 1. Architecture of a distributed NFV Orchestration in a WMN-based Disaster Network

WMN-based nodes but are configured to operate in both channels and thus provide the interconnection of the clusters. Additionally, it should be mentioned that each cluster is defining a multicast group, which each WMN-Node is aware of via a DHCP-related bootstrapping mechanism.

\section{B. VIM Layer}

The VIM Layer defines a Virtualised Infrastructure Manager (VIM) for every NFVI-Node and therefore every node in the WMN. A VIM is according to [4] responsible for the management and controlling of the physical and virtual resources in the NFVI. A VIM is theoretically able to monitor multiple NFVI-Nodes. However, a VIM requires to permanently monitor the resources and virtualisation layer of its NFVI-Nodes for being able to notify an orchestrator at crossing of a defined threshold. By defining a VIM for every NFVI-Node and therefore every WMN node, the required monitoring traffic remains locally on the nodes and is not forwarded through the WMN.

\section{NFVI-Clustered Layer}

The NFVI-Clustered Layer consists of logical clusters on top of the VIM layer. Each cluster defines the area of responsibility for a Cluster-NFVO responsible for orchestrating and managing the resources in its cluster. The Cluster-NFVO realises these aspects by communicating with the VIMs in his area of responsibility. Based on the retrievable information, a Cluster-NFVO can make decisions regarding the orchestration of resources within its cluster. Additionally, the Cluster-NFVOs are responsible for establishing the clusters on the Clustered WMN-based NFVI Layer.

The dynamic formation of the logical clusters in the NFVIClustered Layer, including the integration of a VIM from the VIM Layer into a corresponding cluster as well as the partly monitoring of the resources is a major functionality of the NFV resource advertisement and discovery protocol.

\section{Global NFV-Orchestration Layer}

The Global NFV-Orchestration Layer is used for the intraorchestration communication of the Cluster-NFVOs. The 
TABLE I. Definition and Description of the States of the Protocol State Machine

\begin{tabular}{|c|c|c|}
\hline State of the State Machine & $\begin{array}{c}\text { NFV } \\
\text { component }\end{array}$ & State Description \\
\hline DEPLOYED & undefined & $\begin{array}{l}\text { A new node has been deployed and has joined the network, but its resources have not } \\
\text { yet been integrated into the NFVI. }\end{array}$ \\
\hline ADVERTISED & undefined & $\begin{array}{l}\text { A node has distinguished the resources it intends to provide to the NFVI and advertised } \\
\text { those in the network but has not yet been integrated into the NFVI. }\end{array}$ \\
\hline OBSERVED & VIM & $\begin{array}{l}\text { The node has been successfully integrated by an CNFVO into the NFVI and is aware of } \\
\text { the existence of its responsible CNFVO and is thus acting as a VIM. }\end{array}$ \\
\hline OBSERVATION UNKNOWN & VIM & $\begin{array}{l}\text { A node acting as a VIM has not received any kind of notification from its CNFVO in a } \\
\text { defined time period, which would indicate the existence of the CNFVO. The VIM is } \\
\text { therefore not certain if its responsible CNFVO has failed or not. }\end{array}$ \\
\hline UPDATING LOCAL RESOURCE TABLE & VIM & $\begin{array}{l}\text { The local resources of a VIM have changed, and an individual local resource table is } \\
\text { updated with the corresponding changes. }\end{array}$ \\
\hline STORING GLOBAL RESOURCE TABLE & VIM & $\begin{array}{l}\text { A VIM has received the global resource table of a CNFVO and is storing it for the } \\
\text { purpose of a redundant distributed information storage. }\end{array}$ \\
\hline OBSERVING & CNFVO & $\begin{array}{l}\text { A node is acting as a CNFVO and is observing a set of VIMs in the network. } \\
\text { Additionally, it is listening for nodes which have not yet been integrated into the NFVI. }\end{array}$ \\
\hline UPDATING GLOBAL RESOURCE TABLE & CNFVO & $\begin{array}{l}\text { The resources of a VIM or a set of VIMs a CNFVO is observing have changed. The } \\
\text { CNFVO therefore updates its corresponding global resource table it maintains. }\end{array}$ \\
\hline TAKING/HANDING OVER & CNFVO & $\begin{array}{l}\text { The CNFVO has discovered optimization possibilities or misconfigurations regarding } \\
\text { the clusters of the network. Through a set of algorithms, the CNFVO can distinguish } \\
\text { how to proceed with it. This can either consist of handing over the resources to a new } \\
\text { CNFVO to create a new cluster or by taking over resources from misconfigured nodes. }\end{array}$ \\
\hline SHUTDOWN & undefined & A node has removed his resources from the NFVI and is no longer part of it. \\
\hline
\end{tabular}

communication taking place in this layer consists of the synchronisation of the cluster individual information and the cluster-wide coordination regarding the NFV orchestration.

In case of the initialisation of a new network service or the optimisation of an already deployed network service, a Cluster-NFVO uses the information received from the other Cluster-NFVOs to retrieve a global view over the Clustered WMN-based NFVI Layer. Through this retrievable global view, the Cluster-NFVO will determinate the optimal placement of the network services and functions and notify the corresponding Cluster-NFVOs with the appropriate configuration for their cluster. Additionally, NFV related failures will be handled via the intra-orchestration communication. In case of a local failure that cannot be solved by the corresponding Cluster-NFVO, such as the loss of a large proportion of resources in the local cluster, an event will be send to the other Cluster-NFVOs with regard to the need for immediate intervention for bypassing and solving the failure by redeploying failing VNFs in adjoining clusters.

\section{STATES OF THE NFV RESOURCE ADVERTISEMENT AND DISCOVERY STATE MACHINE}

Each node in the WMN-based disaster network is running and maintaining its individual state machine of the NFV resource advertisement and discovery protocol. A node can only be in one state at a time, while different events can change its state. The possible states of the NFV resource and discovery state machine are listed and described in table I.

The state machine is defining the NFV management functionality of a node in the network. Based on the state a node possesses, its NFV functionality is either undefined, or it acts as a VIM or CNFVO. In case of an undefined functionality, the resources of the node have either not been integrated into the NFVI yet or the node removed its resources from the NFVI. In case of a VIM, the node has advertised the resources it wants to provide to the NFVI in the network and a CNFVO has successfully integrated them into the NFVI. In case of a node acting as a CNFVO, the node is observing existing VIMs and is integrating new VIMs as soon as it has discovered them.

\section{Methods of THE NFV ResourCe AdVERTISEMENT AND DISCOVERY PROTOCOL}

The NFV resource advertisement and discovery protocol is in addition to the state machine defining a set of methods, which are listed and described in table II. Based on the current NFV management functionality a node is providing, it can use a different set of possible methods. The methods are transmitted between the different nodes in the network using either multicast or unicast.

In case of a node acting as a VIM, the methods it is capable to send consists of the initial advertisement of its resources in the network and announcements regarding changes in its locally provided resources. Possible update announcements are comparable to the notifications defined in [8].

The methods provided to a CNFVO are enabling a more extensive set of functionalities. Using these methods, a CNFVO can discover resources in the network which might not have been integrated yet by sending out a discovering message indicating its availability to the VIMs. Additionally, a CNFVO can send out summaries of the resources it maintains and the configuration of the corresponding resources. Through these procedures, VIMs receiving the corresponding multicast message can store the information and thereby enable a redundant distributed information storage. This supports the fault tolerance of the distributed orchestration, since the configurations of the resources an orchestrator maintains are not lost in case of its failure enabling a smooth transition at the initialisation of a new orchestrator instance. Lastly, a CNFVO is capable of handing over resources maintained by a VIM or VIMs to another CNFVO in the network or taking over nodes into its own responsibility.

\section{Behaviour of the Protocol State Machine}

The state machine of the NFV resource advertisement and discovery protocol is shown in figure 2 and describes the connections between the different states which have been defined in section III. The presented methods in section IV are occurring in the transitions from one state to another. 
TABLE II. DEFINITION AND Description of THE EXISTING Protocol Methods

\begin{tabular}{|c|c|c|l|}
\hline Methods of the Protocol & $\begin{array}{c}\text { Initiating NFV } \\
\text { component }\end{array}$ & Transmitted via & Method Description \\
\hline ADVERTISE & undefined & Multicast & $\begin{array}{l}\text { A newly deployed node, which has not been integrated into the NFVI, advertises the } \\
\text { resources it wants to provide to the NFVI by using the ADVERTISE method. }\end{array}$ \\
\hline UPDATE & VIM & Unicast & $\begin{array}{l}\text { An integrated node acting as a VIM uses the UPDATE method to notify a CNFVO } \\
\text { regarding changes in its locally provided resources. }\end{array}$ \\
\hline BYE & VIM & Unicast & $\begin{array}{l}\text { An integrated VIM uses the BYE method to announce its intention of leaving the } \\
\text { network and thus the NFVI towards its CNFVO. }\end{array}$ \\
\hline DISCOVER & CNFVO & Multicast & $\begin{array}{l}\text { A CNFVO can use the DISCOVER message to discover nodes which are currently } \\
\text { not integrated into the NFVI or force other existing nodes to send an update of their } \\
\text { current resources. Nodes receiving this message should either send an ADVERTISE } \\
\text { method or an UPDATE method depending on the content of the DISCOVER. }\end{array}$ \\
\hline OBSERVE & CNFVO & Multicast & $\begin{array}{l}\text { The OBSERVE method can be used by a CNFVO to notify VIMs in the network about } \\
\text { its existence. The message can therefore be interpreted as a keep-alive message. }\end{array}$ \\
\hline SUMMARY & Multicast & $\begin{array}{l}\text { The SUMMARY method can be used by a CNFVO to create a redundant distributed } \\
\text { information storage by forcing receiving VIMs to store the CNFVO's global resource } \\
\text { table, which is transmitted in the corresponding message. In addition, the CNFVO can } \\
\text { attach information for announcing a backup node acting as a backup CNFVO in case } \\
\text { the current CNFVO failing. }\end{array}$ \\
\hline HANDOVER & CNFVO & Multicast & $\begin{array}{l}\text { A CNFVO can force a set of VIMs it maintains to establish a new cluster according } \\
\text { to the provided information in the content of the message. }\end{array}$ \\
\hline TAKEOVER & CNFVO & Multicast & $\begin{array}{l}\text { The TAKEOVER message can be used by a CNFVO to take over a set of nodes into } \\
\text { its responsibility. }\end{array}$ \\
\hline
\end{tabular}

For an improved understanding of the state machine and therefore the protocol itself, the behaviour of the state machine at different scenarios occurring in a dynamic wireless network is explained. While the scenarios are derived from the WMNbased disaster network, the scenarios are not necessarily limited to this kind of network and are also valid for other dynamic wireless networks facing similar issues.

The scenarios used for describing the protocol consists of the deployment of a new node which needs to be integrated into the NFVI of the WMN-based disaster network, the failure and resulting loss of an integrated node, an integrated node leaving the network and the rearrangement of the NFVI resulting from the expansion of the network. Each scenario is explained in different variants, which are introduced in the following.

\section{A. Scenario 1: Deployment of a new node}

Through the deployment of a battery-supplied wireless outdoor router by the rescue helpers, the WMN-based disaster network is either initially started by the first deployed node or further expanded through the deployment of additional nodes. For this reason, two variants of the scenario dealing with the deployment of a new node are described in the following.

\section{1) Variant 1: Deployed node is the first node}

In this variant of the scenario, the deployed node is the first deployed node and is therefore initialising the WMN-based disaster network. Through its deployment, the node is entering the DEPLOYED state of the state machine. In this state it defines the resources it wants to provide to the NFVI and advertises them by sending out the ADVERTISE message, which results in the node entering the ADVERTISED state. This process is valid for all newly deployed nodes. Since the node in this variant of the scenario is the first node, the ADVERTISE method remains unanswered by an existing CNFVO. After the expiration of a definable timer $t$ advertised and the resulting absence of a valid OBSERVE message, the node is sending out a DISCOVER message and enters the OBSERVING state. From this moment on, the node is acting as a CNFVO and is awaiting ADVERTISE messages.

\section{2) Variant 2: Deployed node is not the first node}

In this variant of the scenario, the deployed node is not the first node and a network and the corresponding NFVI have already been established with at least one CNFVO maintaining it. The existence of an CNFVO is ensured due to the previous explained variant of this scenario. As explained in the previous variant, the newly deployed node enters the DEPLOYED state, advertises its resources via an ADVERTISE message and enters the ADVERTISED state.

The ADVERTISE message is received by an existing CNFVO who is responding with a corresponding OBSERVE message and is entering the UPDATING GLOBAL RESOURCE TABLE state. The CNFVO is updating the global resource table it maintains and leaves the state by sending out a SUMMARY message with the updated global resource table. In the message the CNFVO defines additionally the node which will act as a backup CNFVO in case of its failure.

The newly deployed node receiving the valid OBSERVE message is thus entering the OBSERVED state resulting in the node acting as a VIM since its resources have been integrated into the NFVI. Additionally, the following received SUMMARY message is processed by the node by entering the STORING GLOBAL RESOURCE TABLE state, in which the node is storing the global resource table. Through this mechanism, the node stores all current information contained in the summary and thus realises a redundant distributed information storage. This variant of the scenario ends with the VIM entering the OBSERVED state again.

\section{B. Scenario 2: Failure and loss of an integrated node}

Due to the environment of a disaster, an integrated node of the WMN-based disaster network can be destroyed due to spontaneous events such as aftershocks or outbreaking fires. In this case, an affected node is not able to notify other nodes in the network about its destruction. Regarding this possibility and the NFV resource advertisement and discovery protocol, a failing node can either be a CNFVO or a VIM.

1) Variant 1: Failing node is acting as a VIM

In the case of the failing node was acting as a VIM, its neighbouring nodes will identify a change in their local resources since the link to the node has been lost, resulting in the nodes entering the UPDATING LOCAL RESOURCE TABLE state. In this state the nodes are updating their individual local resource table and are returning into the 


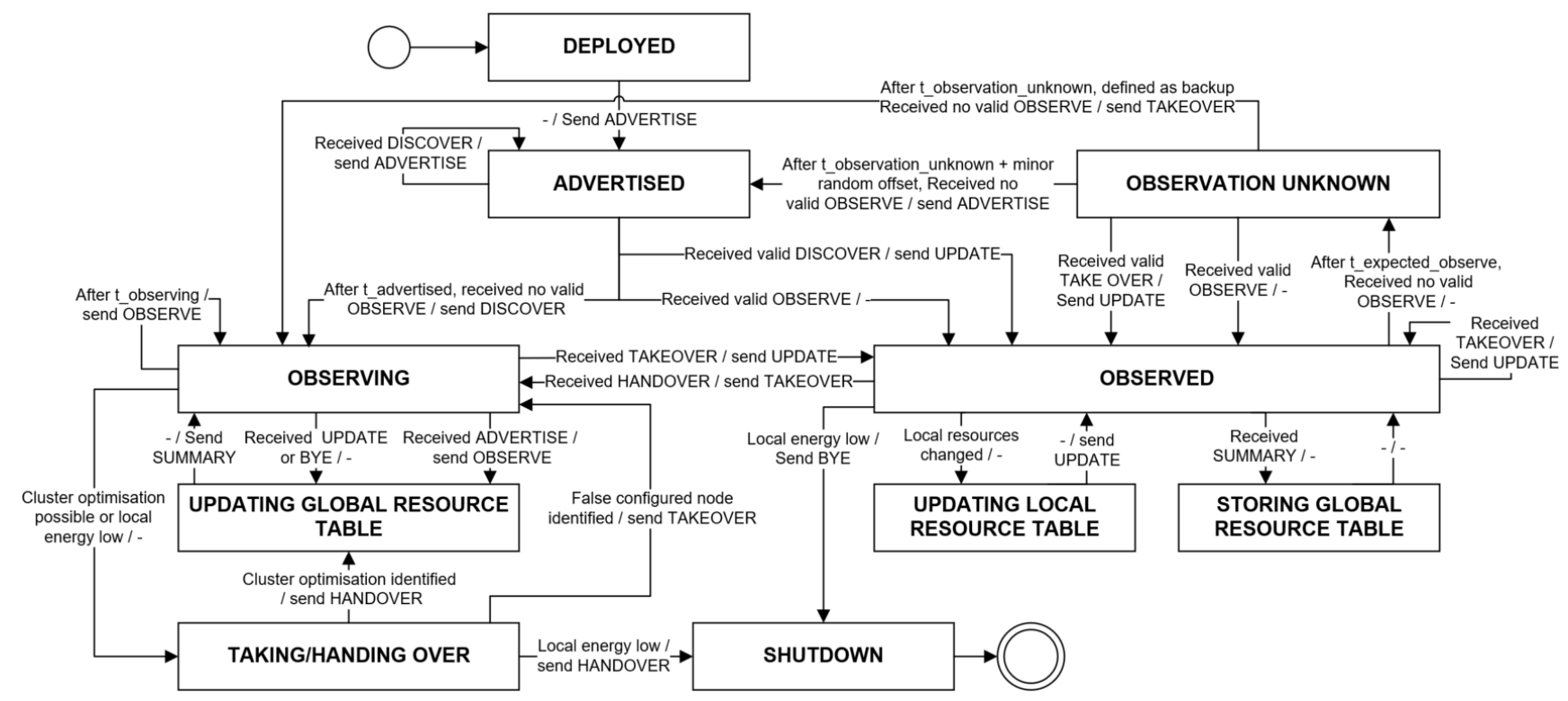

Fig. 2. State Machine of the NFV Resource Advertisement and Discovery Protocol

OBSERVED state by sending an UPDATE message to their CNFVO.

At receiving of the corresponding UPDATE message, the CNFVO, who is currently in the OBSERVING state, is entering the UPDATING GLOBAL RESOURCE TABLE state by removing the failing node from its resource table. Additionally, the CNFVO might react to the failing node by reallocating NFV resources if required. However, this aspect of changes in the actual orchestration of the resources is not part of this publication. After the processing of the UPDATE message, the CNFVO will send out a SUMMARY message with an updated version of its global resource table and returns into the OBSERVING state. Nodes receiving the SUMMARY message will process it as previously explained for realising the redundant distributed information storage.

\section{2) Variant 2: Failing node is acting as a CNFVO}

If the failing node was acting a CNFVO, the resources and VIMs it was responsible for are not maintained nor orchestrated anymore. The nodes, which have been maintained by the CNFVO, will recognise its absence through the missing OBSERVE message a CNFVO is intended to periodically send out based on a timer $t$ observing. After the expiration of a timer $t$ expected_observe which is slightly larger than the t_observing and the absence of a valid OBSERVE message, the nodes are entering the OBSERVATION UNKNOWN state. After the exceeding of an additional timer $t$ observation_unknown without receiving a valid OBSERVE message, the VIM defined as the backup CNFVO by the initial CNFVO is sending out a TAKEOVER message. The TAKEOVER message consists of the VIMs it is aware of through the global resource table it maintained through the received SUMMARY messages. Through the sending of the TAKEOVER message, the backup CNFVO is entering the OBSERVING state and thus becomes the new CNFVO. The nodes in the OBSERVATION UNKNOWN state receiving the corresponding TAKEOVER message will send an UPDATE message towards the new CNFVO and enter the OBSERVED state again.

In case of the backup CNFVO also failing in the time defined by $t$ observation unknown, the remaining VIMs will enter the ADVERTISE state in a random order and send an
ADVERTISE message. The random order is achieved through a minor random offset added to the $t$ observation_unknown timer. The first node entering the OBSERVING state due to a missing OBSERVE message is sending out a DISCOVER message including the VIMs it is aware of from the global resource table it also maintains and becomes the new CNFVO. The receiving VIMs, which are in the ADVERTISED state, send an UPDATE message to the new CNFVO due to the valid received DISCOVER and enter the OBSERVED state. This process compensates the unlikely event that both CNFVO instances fail and thus allows the configuration to be retained.

\section{Scenario 3: Integrated node is leaving the network}

The WMN-based disaster network is constructed from battery-supplied wireless outdoor routers. Even through an optimised energy consumption in the network, the battery of a router will get empty, forcing the node to leave the network and thus the NFVI. Regarding the NFV resource advertisement and discovery protocol the leaving node is either acting as a VIM or as a CNFVO.

\section{1) Variant 1: Leaving node is acting as a VIM}

In the case of the leaving node is acting as a VIM, it will notify its CNFVO regarding its intention via a BYE message and enters the SHUTDOWN state resulting in its resources no longer being part of the NFVI. At receiving of the BYE message, the CNFVO will update its global resource table by entering the UPDATING GLOBAL RESOURCE TABLE and might reallocate NFV resources if required. Through the sending of a SUMMARY message the redundant distributed information storage of the other nodes is updated and the CNFVO continues to listen for other messages by entering the OBSERVING state again.

\section{2) Variant 2: Leaving node is acting as a CNFVO}

A leaving CNFVO needs to ensure that its maintained and orchestrated resources are handed over to another CNFVO before it leaves the network to ensure a continuous orchestration of the NFVI and its resources. For this purpose, a CNFVO with a soon empty battery therefore enters the HANDING OVER state. Through a determination process, the CNFVO defines one of the VIMs, probably the already 
defined backup CNFVO to be future CNFVO. Through the sending of a HANDOVER message, the CNFVO is notifying the VIMs and the future CNFVO about the changes and enters the SHUTDOWN state.

The defined future CNFVO will change into the OBSERVING state by sending out a TAKEOVER message to the VIMs. The VIMs will respond with an UPDATE message and will expect OBSERVE messages from the new CNFVO.

\section{Scenario 4: Rearrangements of the NFVI-Clusters}

The expansion of the WMN-based disaster network through the deployment of additional battery-supplied wireless outdoor routers requires changes in the network infrastructure and the NFVI to achieve evenly distributed clusters in the Clustered WMN-based NFVI Layer and thus the NFVI-Clustered Layer of the architecture of the distributed NFV orchestration (see section II).

In case of a CNFVO identifying the possibility for a cluster optimisation, the corresponding CNFVO enters the TAKING/HANDING OVER state. Based on the optimisation possibility, the CNFVO will either initialise the creation of a new cluster or eliminate possible misconfigurations resulting from a possible network partition happened before.

For the establishment of a new cluster, the CNFVO uses a clustering algorithm which is not part of this publication to define a set of VIMs which will be part of the new cluster. From this set of nodes, the CNFVO also defines the node which will act as the CNFVO of the new cluster. By sending out a HANDOVER message, the corresponding nodes are notified and the sending CNFVO is entering the UPDATING GLOBAL RESOURCE TABLE and removes the notified nodes from its resource table. Afterwards, the changes in the resource table are distributed to the remaining VIMs via a SUMMARY resulting in the CNFVO entering the OBSERVING state again.

The nodes affected by the HANDOVER message will process it according to its content by changing their network configurations. Additionally, the node supposed to act as the future CNFVO sends out a TAKEOVER message to the VIMs listed in the HANDOVER message and enters the OBSERVING state. Through the corresponding forced UPDATE message send by the receiving VIMs, the resource table of the CNFVO of the new cluster is validated.

In case of misconfiguration a CNFVO can identify through the multicast messages in a cluster, it can force the integration by sending a TAKEOVER message to the malfunctioning node. The malfunctioning node, which is either acting as a CNFVO or VIM, accepts the integration by returning an UPDATE message and enters the OBSERVED.

\section{RELATED WORK}

The dynamic formation of an NFVI is considered in [9] in relation to a fog virtualisation environment including mobile and volatile nodes. Nodes moving into the network can either promote themselves as a NFVO or as a VIM. To establish the connection between the logical NFV components, the approach intends to use additional option headers in the IPv6 neighbour discovery and/or advertisement process.

Based on this concept and in relation to the WMN-based disaster network and its distributed NFV orchestration major issues arise. The usage of IPv6, which would be mandatory, cannot be insured in the disaster network. Also, the assignment of a node acting as a NFVO or VIM is predefined based on the capability of a device joining the network, making a permanent availably of an NFVO doubtful.

These drawbacks do not exist in the NFV resource advertisement and discovery protocol since it is universally designed making it independent of the used networking or transport protocol. The transmission protocol only needs to provide a multicast functionality, which in case of a WMN is already provided by the used data link layer protocol. Also, the NFV management functionalities are assigned dynamically to a node by the proposed protocol based on the current network infrastructure. However, the concept of transmitting the information by piggybacking them in an already existing multicast-based protocol such as the routing protocol in the network is promising. This would significantly reduce the overhead of the proposed protocol.

\section{CONCLUSION}

In this publication an NFV resource advertisement and discovery protocol was presented. The protocol enables a distributed orchestration to identify dynamic changes in a network topology and thus the NFVI. While it is designed for the usage in a WMN-based disaster network it is not necessarily limited to this kind of network and could also be used in other dynamic wireless networks which are integrating NFV. The protocol is described using a set of methods and a state machine. The behaviour of the state machine and corresponding message flow of the protocol have been described using a set of scenarios. The scenarios deal with events that affect the network infrastructure and occur in most wireless network. In the future, the protocol will be tested and evaluated in detail by emulations.

\section{ACKNOWLEDGMENT}

The research project VirtO4WMN providing the basis for this publication is partially funded by the Federal Ministry of Education and Research (BMBF) of the Federal Republic of Germany under grant number 13FH018IX6. The authors of this publication are in charge of its content.

\section{REFERENCES}

[1] ITU-T Focus Group on Disaster Relief Systems Network Resilience and Recovery, "Technical report on Telecommunications and Disaster Mitigation," 2013.

[2] A. Lehmann, A. Paguem Tchinda, and U. Trick, "Optimization of wireless disaster network through network virtualization," INC (2016), Frankfurt, pp. 165-170, 2016.

[3] ETSI ISG NFV, "Network Function Virtualisation - An Introduction, Benefits, Enablers, Challenges \& Call for Action," 2012.

[4] ETSI, "Network Function Virtualization (NFV); Management and Orchestration," ETSI GS NFV-MAN 001 V1.1.1, 2014.

[5] M. M. Afsar and M.-H. Tayarani-N, "Clustering in sensor networks: A literature survey," J. Netw. Comput. Appl., vol. 46, pp. 198-226, Nov. 2014.

[6] V. Sucasas, A. Radwan, H. Marques, J. Rodriguez, S. Vahid, and R. Tafazolli, "A survey on clustering techniques for cooperative wireless networks," Ad Hoc Networks, vol. 47, pp. 53-81, Sep. 2016.

[7] G. Frick et al., "Distributed NFV Orchestration in a WMN-Based Disaster Network," in 2018 Tenth International Conference on Ubiquitous and Future Networks (ICUFN), 2018, pp. 168-173.

[8] ETSI, "Network Functions Virtualisation (NFV) Release 3; Management and Orchestration; Or-Vi reference point - Interface and Information Model Specification," ETSI GS NFV-IFA 005 V3.1.1, 2018.

[9] C. J. Bernardos and A. Mourad, "draft-bernandos-intarea-vimdiscovery-01 - IPv6-based discovery and association of Virtualization Infrastructure Manager (VIM) and Network Function Virtualization Orchestrator (NFVO)," IETF INTAREA WG, 2019 